\title{
Mechanics of Streptavidin-Coated Giant Lipid Bilayer Vesicles: A Micropipet Study
}

\author{
Pasut Ratanabanangkoon, ${ }^{\dagger}$ Michael Gropper, ${ }^{\ddagger}$ Rudolf Merkel ${ }^{\S}$ \\ Erich Sackmann, ${ }^{\ddagger}$ and Alice P. Gast*
}

\begin{abstract}
Department of Chemical Engineering, Stanford University, Stanford, California 94305-5025, Department of Biophysics-E22, Technical University of Munich, D-85748 Garching, Germany, Forschungszentrum J ülich, Institut für Schichten und Grenzflächen (ISG-4), D-52425 J ülich, Germany, and Department of Chemical Engineering, Massachusetts I nstitute of Technol ogy, Cambridge, Massachusetts 02139
\end{abstract}

Received J uly 17, 2002. In Final Form: November 4, 2002

\begin{abstract}
Tounderstand the effects of a crystalline protein layer on bilayer properties, we studied the mechanical properties of avidin- and streptavidin-coated giant lipid bilayer vesicles. The giant vesicles $(20-60 \mu \mathrm{m})$ are made from a mixture of SOPC and biotinylated phospholipids via el ectroformation. Using micropipet manipulation, we showed that the presence of a monomolecular layer of noncrystalline avidin on the vesicle surface increases the membrane bending rigidity but does not significantly alter the elastic area expansion modulus of the vesicle. When the vesicles were coated with streptavidin, the protein crystallizes on thebilayer surface, resulting in a rigi d polycrystallinemembrane. These vesicles display unique roughened spherical or prolateel lipsoidal shapes, depending on the differences in crystal morphol ogies. U pon aspiration with micropi pets, the vesicles first showed rapid permanent deformation at low strain, foll owed by a sl ower viscoelastic response above a certain threshold. Despite their extremely rigid appearance, the existence of a polycrystalline shell does not increase the toughness of streptavidin-coated vesicles above that of uncoated vesicles. The origin of these properties can be traced to the unique ligand-receptor interactions between streptavidin and bi otinylated phospholipids in the bilayer membrane. The findings offer greater understandings of compl ex phenomena invol ving crystalline protein layers on the surface of cell membranes, in addition to providing information for the devel opment of various appl ications involving theimmobilization of functionalized molecules on lipid bilayer substrates.
\end{abstract}

\section{Introduction}

Properties of lipid bilayer membranes and membraneprotein complexes have been of great interest and the subject of numerous studies due to their physical and biological importance and potential applications. ${ }^{1-3}$ In particular, they serveas simplified model stothecomplex and inhomogeneous properties of actual cell membranes. The possibility of incorporating molecules into the membrane to systematically alter its composition allows the separate study of individual membrane components and has led to significant understanding of a number of membraneproperties and cellular functions. ${ }^{4-8}$ Theability to vary bilayer composition also allows custom tailoring of bilayer surfaces to suit various applications, such as

* To whom correspondence may be addressed at the Massachusetts Institute of Technology. Phone: (617) 253-1403. FAX: (617)-253-8388. E-mail: gast@mit.edu.

† Stanford University. E-mail: pasut@eland.stanford.edu.

¥ Technical University of Munich. E-mail: Michael Gropper, michael.gropper@web.de; Rudolf Merkel, r.merkel@fz-juelich.de.

$\S$ Institut für Schichten und Grenzflächen. E-mail: r.merkel@ fz-juelich.de.

(1) Sackmann, E. Science 1996, 271, 43-48.

(2) Luisi, P. L.; Walde, P. Giant Vesicles; J ohn Wiley \& Sons, Ltd: New York, 2000; Vol. 6.

(3) Cevc, G. Phospholipids handbook; Marcel Dekker: New York, 1993.

(4) Dietrich, C.; Yang, B.; Fujiwara, T.; Kusumi, A.; J acobson, K. Biophys. J . 2002, 82, 274-284.

(5) Hackl, W.; Barmann, M.; Sackmann, E. Phys. Rev. Lett. 1998, 80 , $1786-1789$

(6) Schindler, M.; Osborn, M. J .; Koppel, D. E. Nature 1980, 283, 346-350.

(7) Parlati, F.: McNew, J. A.; Fukuda, R.; Miller, R.; Sollner, T. H. Rothman, J. E. Nature 2000, 407, 194-198.

(8) Needham, D.; Macintosh, T. J .; Lasic, D. D. Biochim. Biophys. Acta 1992, 1108, 40-48. the attachment of biomolecules to inorganic surfaces. Through Langmuir-Blodgett ${ }^{9,10}$ or vesicle fusion ${ }^{11-13}$ techniques, lipid bilayers are used to create fluid layers on solid-liquid interfaces for the attachment of other functionalized molecules. ${ }^{1,14} \mathrm{~F}$ unctionalization of the bilayer surface often involves thebinding of an intermediate monomolecular layer of protein which itself acts as either a receptor or a support for the attachment of other ligands and biomolecules. In some biosensor applications, the formation of ordered arrays of functionalized molecules on supported bilayers is desired. This can be achieved by crystallizing an underlying protein layer into an ordered array. While it is clear that certain alterations made to the bilayer membrane can affect its properties and thus its uses, the change in the bilayer properties due to the presence of an outer crystallineprotein layer has not been thoroughly investigated.

Through the use of giant vesicles as model bilayers, we studied the effect of a surface crystalline protein layer on the mechanical properties of themembrane. In particular, we used micropipet aspiration to probe the membrane responses and material properties of vesicles that exhibited (a) no coating, (b) a crystalline streptavidin layer at the surface, or (c) a monolayer of homogeneous yet noncrystallizable protein avidin.

(9) Blodgett, K. B. J . Am. Chem. Soc. 1935, 57, 1007

(10) Langmuir, I. Trans. Far. Soc. 1920, 15, 62

(11) Kalb, E.; Frey, S.; Tamm, L. K. Biochim. Biophys. Acta 1992 $1103,307-316$.

(12) Tamm, L. K.; McConnell, H. M. Biophys. J . 1985, 47, 105-113.

(13) Brian, A. A.; McConnell, H. M. Proc. Natl. Acad. Sci. U.S.A. 1984, 81, 6159-6163.

(14) Zasadzinski, J . A.; Viswanathan, R.; Madsen, L.; Garnaes, J .; Schwartz, D. K. Science 1994, 263, 1726-1733. 
Streptavidin, a tetramericglobular protein that has an unusually high binding affinity $\left(10^{-15} \mathrm{M}\right)^{15}$ for biotin, is commonly used as a linker to immobilize various biotinylated molecules to surfaces. Streptavidin can crystallizeintohighly ordered arrays on various two-dimensional (2D) surface such as lipid monolayers, ${ }^{16-23}$ lipid bilayers at the solid-liquid interface, ${ }^{24}$ lipid nanotubes, ${ }^{25,26}$ and, recently, giant bilayer vesicles. ${ }^{27}$ Thepresence of two pairs of biotin-binding sites on opposing sides of each streptavidin molecule, along with its high affinity for biotin, results in extensive use as a mol ecular linker in various applications. ${ }^{28-30}$

This work aims toward a fundamental understanding of self-assembly processes in biological systems. It has been known that hundreds of species of bacteria and archea found in various environments contain a crystal line layer of surface proteins at thecell surface called s-layers. Specific functions of s-layer proteins vary with different bacterial species and includenutrient transport, adhesion sites, and protective functions, especially in archaea lacking a rigid wall layer. ${ }^{31,32}$ These proteins self-assemble onto cell surfaces via noncovalent forces such as electrostatic and hydrophobic interactions. In this study the streptavidin-biotin receptor-ligand interaction system can also be made to produce crystall line layers imparting somemechanical strength tothebilayer. Attempts tostudy the properties of the natural crystalline layer on actual cel Is are often complicated by other underlying structural components such as peptidoglycan layers in addition to the complex cytoplasmic composition and the minutesize of bacteria. Crystallizing proteins on giant bilayer vesicles provides us with a similar, well-defined system that is easy to study and manipulate. The unique properties of these structures greatly facilitate both a fundamental understanding of molecular self-assembly and proteinmembrane interactions and potential applications involving the immobilization of functionalized molecules on bilayer membranes.

(15) Bayer, E. A.; Benhur, H.; Wilchek, M. Methods Enzymol. 1990, 184, 80-89.

(16) Darst, S. A.; Ahlers, M.; Meller, P. H.; Kubalek, E. W.; Blankenburg, R.; Ribi, H. O.; Ringsdorf, H.; Kornberg, R. D. Biophys. J. 1991, 59, 387-396.

(17) Frey, W.; Schief, W. R.; Pack, D. W.; Chen, C. T.; Chilkoti, A.; Stayton, P.; Vogel, V.; Arnold, F. H. Proc. Natl. Acad. Sci. U.S.A. 1996, 93, 4937-4941.

(18) Hemming S. A. Bochkarev, A.; Darst, S. A. Kornberg, R. D : Ala, P.; Yang, D. S. C.; Edwards, A. M.J . Mol . Biol. 1995, 246, 308-316. (19) Ku, A. C.; Darst, S. A.; Kornberg, R. D.; Robertson, C. R.; Gast,

A. P. Langmuir 1992, 8, 2357-2360.

(20) Wang, S.-W.; Poglitsch, C. L.; Yatcilla, M. T.; Robertson, C. R.; Gast, A. P. Langmuir 1997, 13, 5794-5798.

(21) Wang, S.-W.; Robertson, C. R.; Gast, A. P. Langmuir 1999, 15, $1541-1548$

(22) Scheuring, S.; Müller, D. J .; Ringler, P.; Heymann, J . B.; Engel, A. J. Microsc. 1999, 193, 28-35.

(23) Farah, S.J .; Wang, S. W.; Chang, W. H.; Robertson, C. R.; Gast,

A. P. Langmuir 2001, 17, 5731-5735.

(24) Calvert, T. L.; Leckband, D. Langmuir 1997, 13, 6737-6745. (25) Wilson-Kubalek, E. M.; Brown, R. E.; Celia, H.; Milligan, R. A.

Proc. Natl. Acad. Sci. U.S.A. 1998, 95, 8040-8045.

(26) Ringler, P.; Müller, W.; Ringsdorf, H.; Brisson, A. Chem. Eur. J . 1997, 3, 620-625.

(27) Ratanabanangkoon, P.; Gropper, M.; Merkel, R.; Sackmann, E.; Gast, A. P. Langmuir 2002, 18, 4270-4276.

(28) McLean, M. A.; Stayton, P. S.; Sligar, S. G. Anal. Chem. 1993, $65,2676-2678$ 1209.

(30) Samuelson, L.A. Wiley: B. Kaplan, D.L., Sengupt

(30) Samuelson, L. A.; Wiley: B.; Kaplan, D. L.; Sengupta, S.; Kamath,
M.: Lim, J. O.; Cazeca, M.; Kumar, J.; Marx, K. A.; Tripathy, S. K. J Int. Mater. Syst. Struct. 1994, 5, 305-310.

(31) Sleytr, U. B.; Messner, P.; Pum, D.; Sára, M. Crystallinebacterial cell surface proteins; Academic Press: Austin, TX, 1996.

(32) Sleytr, U. B.; Messner, P.; Pum, D.; Sára, M. Crystallinebacterial cell surface layers; Springer-Verlag: Berlin, Germany, 1988.

\section{Material and Methods}

Preparation of Vesicles. Giant unilamellar vesicles (GUVs) weremadeby el ectroformation. ${ }^{33}$ Thelipids SOPC (1-stear oyl-2-ol eoyl-sn-glycero-3-phosphocholine) from Avanti Polar Lipids (Alabaster, AL) and biotin-X-DHPE (N-((6-(biotinoyl)amino)hexanoyl)-1,2-dihexadecanoyl-snglycero-3-phosphoethanolamine, triethylammonium salt) from Molecular Probes (Eugene, OR) were mixed in a weight ratio of 10:1 and dissolved in chloroform (DAB9 grade, Sigma-Aldrich Chemie, Deisenhofen, Germany) to a total concentration of $1 \mathrm{mg} / \mathrm{mL}$. Solutions were kept at $-70{ }^{\circ} \mathrm{C}$ until use. Approximately $50 \mu \mathrm{L}$ of this solution were spread on two indium tin oxide coated glass plates, which measured $6 \mathrm{~cm} \times 6 \mathrm{~cm} \times 0.1 \mathrm{~cm}$. Li pid-coated glass plates were dried in a vacuum chamber for at least $2 \mathrm{~h}$ toremovethesolvent. Subsequently, theglass plates were placed in a swelling chamber with the coated sides facing each other. Both plates were held at a distance of $0.5 \mathrm{~mm}$ by a thin Teflon spacer. The vesicles wereswollen in $2 \mathrm{~mL}$ of a $610 \mathrm{mOsm}$ sucrose solution. After filling the chamber, a sinusoidal $A C$ voltage of $1 \mathrm{~V}$ amplitude and $10 \mathrm{~Hz}$ frequency was applied for $2 \mathrm{~h}$ at room temperature. This procedure has a high yield of GUVs of radii between 10 and $60 \mu \mathrm{m}$.

Protein Labeling. Streptavidin and Avidin were purchased from Prozymel nc. (San Leandro, CA). A portion of each protein was labeled with Texas Red succinimidyl ester (single isomer) (Molecular Probes, Eugene, OR) according to the supplied protocol, with minor modifications. Briefly, the proteins were dissolved in $100 \mathrm{mM}$ sodium bicarbonate buffer, $\mathrm{pH}$ 8.3, to a concentration of $2 \mathrm{mg} / \mathrm{mL}$. The fluorescent dye was then dissolved in reagent grade DMSO (Sigma, St. Louis, MO) to a concentration of $10 \mathrm{mg} / \mathrm{mL}$ and added to the protein solution according to the desired labeling ratio. The mixture was stirred in the dark at room temperature for $2 \mathrm{~h}$. Thelabeled protein was separated from theunreacted dye by passing through a size exclusion column packed with Sephadex G25 (Sigma, St. Louis, MO) and eluted with $50 \mathrm{mM}$ sodium phosphate, $\mathrm{pH}$ 7.0. The Texas Red dye (TR) was used with lipid bilayers due to its high stability against photobleaching. The final degree of labeling, one dye molecule per 10 protein molecules, was determined by spectrophotometry.

Crystal Formation on Vesicle Surface. To grow streptavidin crystals on the surface of SOPC/Biotin-XDHPE vesicles, $80 \mu \mathrm{L}$ of the newly el ectroformed vesicles were added to $420 \mu \mathrm{L}$ of protein solution. The protein solution consists of either pure streptavidin or a streptavidin/TR-avidin mixturein $608 \mathrm{mOsm}$ sucroseat an overall concentration of $10-30 \mu \mathrm{g} / \mathrm{mL}$. Pure $608 \mathrm{mOsm}$ sucrose solution has a pH of approximately 5; we controlled the $\mathrm{pH}$ range of the protein solution from 4 to 7.5 by adding $0.1-2 \mu \mathrm{L}$ of either $500 \mathrm{mM}$ Tris, $\mathrm{pH} 10$, or $500 \mathrm{mM}$ MES, $\mathrm{pH}$ 3.15. MES and Tris were chosen despite their suboptimal buffering capacity, since they provide far greater stability to the vesicles than all other types of buffers tested. Care was taken to keep the total buffer concentration in the protein solution under $2 \mathrm{mM}$, as higher ionic concentrations reduce vesicle stability. The vesicl es were incubated in the protein solution for at least $3 \mathrm{~h}$ at room temperature before observation.

Micromanipulation. We manipulate vesicles with micropi pets with an inner diameter between 4 and $10 \mu \mathrm{m}$. To this end, glass capillaries (Hilgenberg, Malsfeld, Germany) with an outer diameter of $1 \mathrm{~mm}$ and an inner

(33) Angelova, M. I.; Soléau, F .; Méléard, P.; Faucon, J . F .; Bothorel, P. Prog. Colloid Polym. Sci. 1992, 89, 127-131. 
diameter of $0.5 \mathrm{~mm}$ were formed into needles with long tips using a pipet puller (Sutter, San Rafael, CA). The tips were broken with a microforge to form an open and even end with a diameter of the necessary size. ${ }^{34}$ Theinner diameter of the pi pets was measured by insertion of goldcoated glass needles that were calibrated by scanning electron microscopy (SE M). Thelength of theneedleinside the pipet was measured with the light microscope; its radius at this point was known from the SEM pictures. With this procedure the inner diameter of the pipets was determined to an accuracy of at least $0.1 \mu \mathrm{m}$.

Themicropi pets werefilled with double deionized water by using a Microfil plastic cannula (World Precision Instruments, Berlin, Germany) and inserted into an injection holder ( $\mathrm{HI}-7$ Narishige I nternational, London, UK). The holder was placed into a three-axis water hydraulicmicromanipulator ( $\mathrm{MHW}-3, \mathrm{Narishige)}$ that was attached to a coarse manipulator (MMN-1, Narishige). The hol der is connected to a hydraulic system that all ows theapplication of suction pressuretothe pi pet. ${ }^{34}$ Pressures up to $8 \mathrm{kPa}$ were measured with a $\mathrm{U}$ tube to an accuracy of $50 \mathrm{~Pa}$.

The micromanipulation chamber was formed by attaching a glass cover slip and 2-mm-thick spacers to a pl exiglass block with vacuum grease(Siliconfett medium, Wacker Chemie GmbH, Munich, Germany). To control temperature, the chamber was cooled by circulating thermostated water through channels in the Plexiglas block. The chamber was open on two sides to allow easy access for the micropipet. It was filled with $500 \mu \mathrm{L}$ of glucose solution with $1 \mathrm{mg} / \mathrm{mL}$ bovine serum albumin (BSA) to prevent the vesicles from adhering to glass surfaces. The experiments were performed on the stage of an inverted light microscope (Axiovert 135TV, Carl Zeiss, J ena, Germany) equipped with a LD-Achroplan 40x 0,60 Korr lens (Carl Zeiss). F or fluorescence microscopy and differential interference contrast (DIC) microscopy, the appropriate optical accessories (filters and Wollaston prisms) recommended by the microscope manufacturer were used. I mages weretaken with a CCD camera C240075I controlled by an Argus 20 image processor (Hamamatsu Photonics, Hamamatsu City, J apan). The latter was connected to the SCSI controller of a PC. Still images weretaken by direct transfer of digital data from theimage processor tothe PC. M ovies wereacquired by data storage on videotape (PanasonicAG-7350 S-VHS recorder, Panasonic, Matsushita ElectricI ndustrial Corp., Osaka, J apan) and subsequent digitization using a Power Macintosh 8600/250 computer (Apple Computer, Cupertino, CA) equipped with a frame-grabber card LG3 (Scion Corp., Frederick, MD). The programs NIH I mage 1.58 VDM and Image (W. Rasband, National Institutes of Health, Bethesda, MD) were used for image processing and measurements.

To determine the elastic area expansion modulus, we followed Kwok and Evans. ${ }^{35}$ Upon aspiration of a fluid phase lipid vesicle, its membrane is tensed. The mechanical surface tension, $T$, is given by

$$
\mathrm{T}=\frac{1}{2} \Delta \mathrm{P} \frac{\mathrm{R}_{\mathrm{P}} \mathrm{R}_{0}}{\mathrm{R}_{0}-\mathrm{R}_{\mathrm{P}}}
$$

where $R_{P}$ and $R_{0}$ denote the radii of the pipet and the vesicle, respectively, and $\Delta \mathrm{P}$ is the applied aspiration pressure. F or uncoated vesicles from phosphol ipids in the fluid phase, the membrane response shows two different

(34) Evans, E. A. Methods Enzymol. 1989, 173, 3-35

(35) Kwok, R.; Evans, E. Biophys. J . 1981, 35, 637-652. regimes. At membranetensions bel ow approximately 0.1 $\mathrm{mN} / \mathrm{m}$, membrane bending is probed, i.e., here the reduction of thermally driven undulations is the most important process. The elastic increase of the bare membrane surface dominates at higher tensions, ${ }^{36}$ producing a linear dependence of therelativearea expansion $\alpha$ on the membrane tension.

$$
\mathrm{T}=\mathrm{T}_{0}+\mathrm{K} \alpha
$$

Here, $\mathrm{K}$ is theelasticarea expansion modul us. Therelative area expansion, $\alpha$, can becalculated with theradii $R_{p}$ and $\mathrm{R}_{0}$ and the change of the projection length, $\Delta \mathrm{L}$, inside the pipet. ${ }^{36}$

$$
\alpha=\frac{\Delta A}{A_{0}} \cong \frac{2 \pi}{A_{0}} R_{P}\left(1-\frac{R_{P}}{R_{0}}\right) \Delta L
$$

Wemeasured the relativearea expansion, $\alpha$, for a number of different membrane tensions for each vesicle. The data where reversible, i.e., increasing and decreasing the membrane tension yielded identical results. The actual area expansion moduli were calculated from fits to linear plots of $\alpha$ versus T.

Vesicles were aspirated by pipets with inner diameters between 5 and $6 \mu \mathrm{m}$ with preset suction pressures between 300 and $3000 \mathrm{~Pa}$ for pressurepulseexperiments. Thetime course of aspiration and the subsequent motion of the aspirated part of the vesicle into the pi pet were recorded with a time resolution of 25 frames per second.

Membrane Mobility. For a qualitative investigation of the lateral mobility of the membrane components, vesicles were prepared such that they either contained fluorescently labeled lipid (1 mol \% NBD-PC from Molecular Probes) or were coated with fluorescently label ed streptavidin (Texas-Red label, see above). These vesicles wereobserved by using a standard fluorescence mi croscope (Zeiss Axiovert 200, equipped with a PIan-N eofluar 100x/ 1.3oil lens, a 100-W mercury arclamp, and theappropriate filter sets, all supplied by Carl Zeiss, J ena, Germany; an ORCA ER digital CCD camera manufactured by Hamamatsu Photonics, Hamamatsu City, J apan was used for observation). First, an image of the entire vesicle was recorded, theillumination aperturewas then closed down and the light intensity was increased by a factor of 12 . This resulted in rapid bleaching of the fluor ophores within the illuminated area. Subsequent redistribution of the fluorescent molecules was followed with sequential fluorescence micrographs of the entire vesicle at a reduced excitation light intensity.

Confocal Microscopy. The vesicles coated with the streptavidin/TR-avidin mixture were transferred to a viewing cell filled with 618 mOsm glucose solution containing $1 \mathrm{mg} / \mathrm{mL}$ BSA. The lower density of surrounding glucose solution causes the sucrose-filled vesicles to sink to the bottom of the cell and remain still during observation.

The confocal laser scanning microscope consists of a scanning unit (N oran, Middleton, WI) attached to a Zeiss Axiovert 100 TV (Zeiss, Germany). The vesicles were scanned with a laser passing through a 568/590LP excitation/emission filter set and viewed with an LDAchroplan $40 \times 0.60 \mathrm{~K}$ orr lens (Carl Zeiss). To prevent superpositi on of images from oppositesides of the vesicle, image slices of only the upper half of the vesicles were taken at $0.5-1-\mu \mathrm{m}$ intervals and a three-dimensional reconstruction was created by using the Intervision 


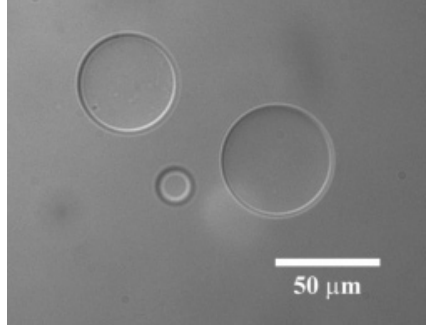

Figure 1. DIC Microscopy image of bareSOPC/Biotin-X-DHPE vesicles.
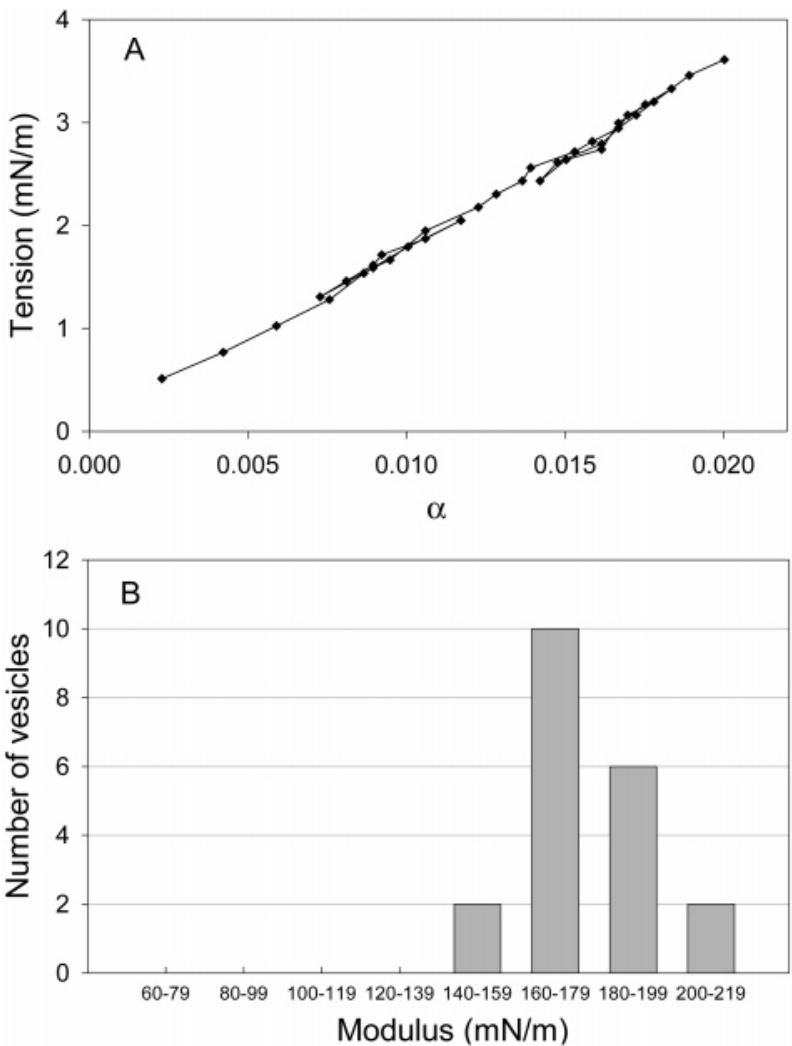

Figure 2. (A) Typical membrane tension vs fractional area expansion $(\alpha)$ data for bare SOPC/Biotin-X-DHPE vesicles. (B) Distribution of area expansion moduli. The average value is $177 \mathrm{mN} / \mathrm{m}$ with a standard deviation of $15 \mathrm{mN} / \mathrm{m}(\mathrm{n}=20)$.

software package provided by N oran on a Silicon Graphics Indy II computer (SGI Inc., Mountain View, CA).

\section{Results}

Bare SOPC/Biotin-X-DHPE Vesicles. The electroformation method described aboveyiel ds numerous stable, round vesicles between 10 and $60 \mu \mathrm{m}$ as shown in Figure 1. At higher external glucose concentrations, the vesicles are deflated and loose their spherical shape due to the large excess area on the surface. ${ }^{37}$ Thermal fluctuations on the membrane can clearly be seen when the vesicles are viewed under these conditions.

We examined the area expansion moduli of these vesicles. The projection changes rapidly and smoothly in response to the applied tension as shown in Figure 2A. The points are connected to show the responses to sequential changes in the pipet suction pressure. The superimposition of the increasing and decreasing suction pressure data onto a single line indicates the vesicle's elastic response to area dilation. The average area

(37) Lipowsky, R. Nature 1991, 349, 475-481.
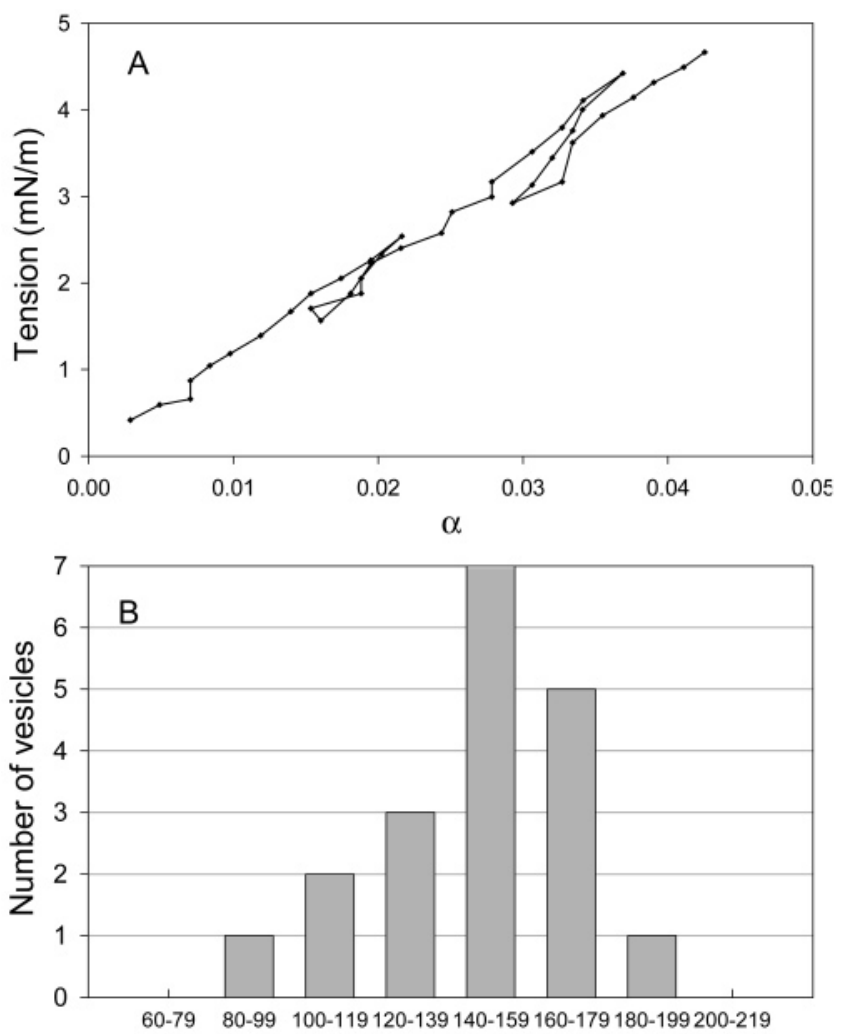

Modulus $(\mathrm{mN} / \mathrm{m})$

Figure 3. (A) Typical membrane tension vs fractional area expansion $(\alpha)$ measurement for avidin-coated vesicles. (B) Distribution of area expansion moduli. The average value is $146 \mathrm{mN} / \mathrm{m}$ with a standard deviation of $27 \mathrm{mN} / \mathrm{m}(\mathrm{n}=19)$.

expansion modulus of $177 \pm 15 \mathrm{mN} / \mathrm{m}(\mathrm{n}=20)$ was in closeagreement toliteraturevalues for pureSOPC vesicles of approximately $180 \mathrm{mN} / \mathrm{m} .36,38,39$

Figure $2 \mathrm{~B}$ shows the relatively narrow distribution of the area expansion moduli of bare vesicles. The distribution of moduli is plotted on thesamescal eas thoseobtained for avidin and streptavidin-coated vesicles presented below. The bare vesicles rupture very rapidly; the entire rupturing process generally takes less than one second and can becharacterized by themembranevanishing into the pipet.

Avidin-Coated SOPC/Biotin-X-DHPE Vesicles. After incubation with avidin, the vesicles were still smooth, spherical, and devoid of creases and folds; however, membrane undulations were no longer visible even when the vesicles were placed in a significantly higher osmolarity glucose solution (839 mOsm). This implies a significant increase in membrane bending stiffness. Due to the high isoelectric point ( $\mathrm{pl}$ ) of avidin ( $\mathrm{pl} \sim 10$ ), the avidin-coated vesicles adhered to the gl ass surfaces. The effect was greatly reduced, though not completely el iminated, through the addition of higher concentrations of BSA ( $2 \mathrm{mg} / \mathrm{mL}$ ) into the solution. Micropipet aspiration of vesicles with fluorescently label ed avidin cl early showed the presence of protein on the aspirated portion of the membrane, indicating that the membrane and protein layer is aspirated together. Their area expansion moduli are similar to those of bare vesicles, as shown in a typical data set (Figure $3 \mathrm{~A})$. Theaverage area expansion modulus of avidin-coated vesicles is $146 \pm 27 \mathrm{mN} / \mathrm{m}(\mathrm{n}=19)$. The

(38) Hianik, T.; Passechnik, V.I. Bilayer Lipid Membranes: Structure and Mechanical Properties; Kluwer AcademicPublishers: Boston, 1995.

(39) Needham, D.; Nunn, R. S. Biophys. J . 1990, 58, 997-1009. 


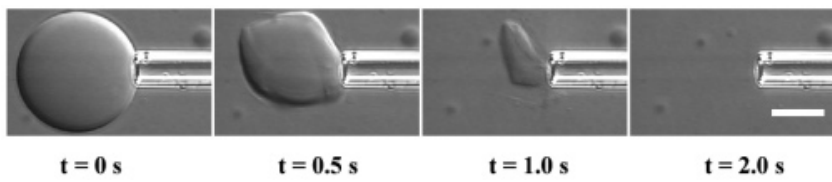

Figure 4. DIC image sequence of a typical rupturing process of avidin-coated vesicles, showing a slow "deflation" behavior. Scale bar represents $10 \mu \mathrm{m}$.

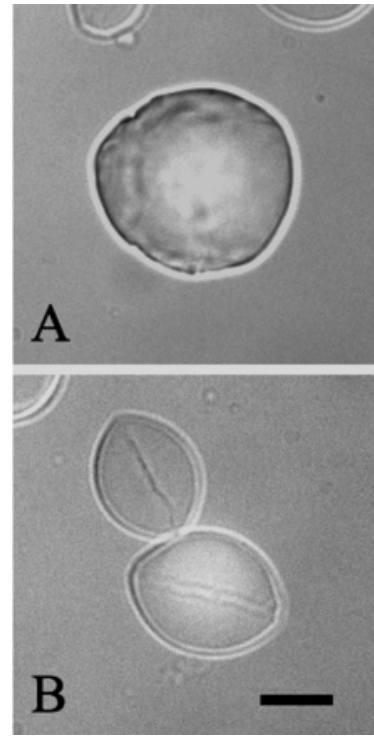

Figure 5. DIC microscopy images of two typical mor phologies of streptavidin-coated vesicles. (A) Spherical vesicles with rough surfaces. (B) F ootball-shaped el lipsoids havesmoother surfaces with characteristic ridges parallel to the major axis. Scale bar is $20 \mu \mathrm{m}$.

comparatively large scatter of the data is caused by residual adhesion of the membrane to the pipet.

Despite their similarity to bare vesicles, avidin-coated vesicles show a dramatically different rupturing behavior. Instead of therapid instantaneous rupturenormally seen, the coated vesicles break with a much slower deflation process (Figure 4). Although the actual deflation time depends on the applied suction pressure and vesiclesize, typical time scales for the rupturing process are on the order of seconds. Theslow deflation and thelack of visible rigid edges and creases are indicative of the presence of a protein shell, which is most likely fluid.

Streptavidin-Coated SOPC/Biotin-X-DHPE Vesicles. When the vesicles were incubated with pure streptavidin, they were no longer smooth and spherical. Instead, the vesicles were more rigid with numerous creases and facets on the surface, indicativeof the presence of a polycrystalline shell. Thermal undulations were no longer seen. Unlike avidin-coated vesicles, the streptavidin-coated vesicles remained unperturbed by strong nudges with the mi cropipet tip. In general, we observed two typical vesicle morphologies shown in Figure 5.

The two morphologies coexist within the same sample, with an increasing fraction of prol ate ellipsoids at higher $\mathrm{pH} .{ }^{27}$ Both morphol ogies havesimilar el astic behavior and properties.

To verify that streptavidin crystals are present on the surface of thesegiant vesicles, we performed laser scanning confocal microscopy studies on vesicles coated with a mixture of streptavidin and TR-avidin. Figure 6 shows images of vesicl es coated with approximately a 1:1 mixture of streptavidin and TR-avidin. Avidin was included to dilutethestreptavidin at thesurface, allowing uninhibited growth of larger crystalline domains. The streptavidin crystals can be seen in fluorescence microscopy because the labeled avidin is excluded from the crystals.

Unlike the vesicles in Figure 5, the vesicles in Figure 6 remained relatively smooth and round due to the low crystallitedensity on thesurface in the presence of avidin. The crystal line area increases with the amount of streptavidin on the surface, and covers the entire vesicle surface when $100 \%$ streptavidin is used.

Streptavidin vesicles also ruptureby slow deflation with a time scale on the order of seconds. One difference from the case of avidin-coated vesicles is the presence of sharp ridges during the crumpling process as shown in Figure 7.

Another property uniquetostreptavidin-coated vesicles is permanent deformation. Figure 8 shows an image sequence of a streptavidin-coated vesicle being aspirated into a micropipet and quickly rel eased. The deformati on did not recover within the observation time of $1.5 \mathrm{~h}$, characteristic of a plastic behavior.

Streptavidin-coated vesicles also show shape changes when aspirated by using a micropipet. The vesicle gradually changes from its rough spherical shapeintoa smoother form (Figure 9).

A representativevesicleresponsetoa stepwise increase in suction pressure is shown in Figure 10. At tensions up to approximately $2 \mathrm{mN} / \mathrm{m}$, the vesicle is permanently deformed, and the projections do not retract when suction pressure is reduced. Friction forces between the vesicle and the glass wall of the pipet were not the cause of this behavior, as the vesicle and its permanently deformed projection easily slides out of the pipet when the suction pressure is removed as shown in Figure 8. In addition, the membrane response in this low-pressure regime is almost instantaneous. When tensions above approximately $2 \mathrm{mN} / \mathrm{m}$ are applied, additional deformations are nolonger permanent. I nstead, thevesicleprojection shows a slower exponential viscoelastic response, and the projection length increases proportionally to the suction pressure (Figure 10). It is worth noting that all the streptavidin-coated vesicles studied using micropipet aspiration exhibited the permanent deformation-viscoelastic transition regardless of vesicle morphology. At these tension values, the applied strain likely involves the direct area dilation of the vesicles. Despite the polycrystalline nature of the composite protein-lipid membrane, which likely imposes an anisotropic strain to the vesicle, the membrane response to stepwise increase in tension is linear.

Using an analysis similar to that of Discher et al. on cross-linked polymer vesicles, ${ }^{40}$ the slope of plots of nominal tension vs dimensionless projection length $\mathrm{x}=$ $\mathrm{L} / \mathrm{R}_{\mathrm{p}}$ can berel ated tothe el asticarea expansion moduli, ${ }^{41}$ resulting in the expression $\mathrm{K} \approx 32 \mathrm{~d} \tau / \mathrm{dx}$ for $\mathrm{R}_{0} / \mathrm{R}_{\mathrm{p}}$ values of 2.5-4.1. This yields $\mathrm{K}=68 \pm 27 \mathrm{mN} / \mathrm{m}$. In general, once deformed past $2 \mathrm{mN} / \mathrm{m}$, the streptavidin-coated vesicles showed much less resistanceagainst area dilation than either bare or avidin-coated vesicles. Despite the rigid appearance, the vesicles rupture at tensions of 4.9 $\pm 1.3 \mathrm{mN} / \mathrm{m}(\mathrm{n}=15)$, within error to that obtained from uncoated pure SOPC vesicles $(5.7 \pm 0.2 \mathrm{mN} / \mathrm{m}) .{ }^{39} \mathrm{Not}$ surprisingly, the distribution of the values is broad due to large variations in the structure of the polycrystalline shell.

To study the vesicle response to sudden changes in suction pressure, a series of alternating pressure pulses

(40) Discher, B. M.; Bermudez, H.; Hammer, D. A.; Discher, D. E. J . Phys. Chem. B 2002, 106, 2848-2854.

(41) Evans, E. A.; Skalak, R. Mechanics and Thermodynamics of Biomembranes; CRC Press: Boca Raton, 1980. 

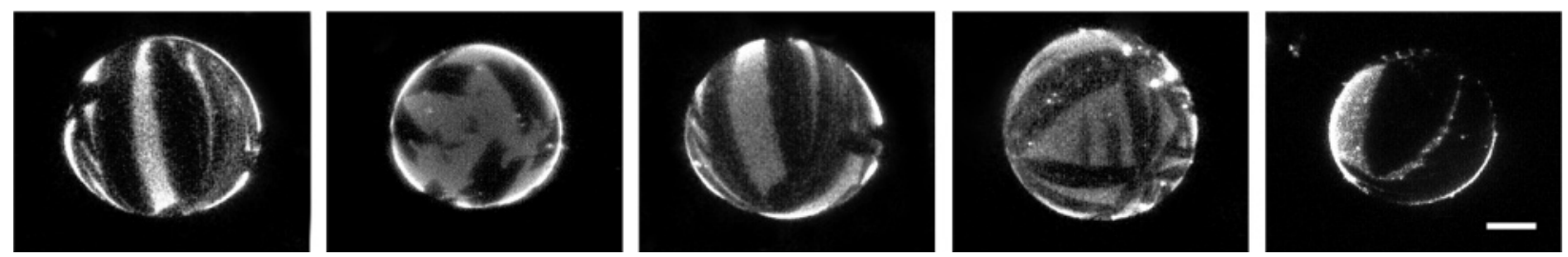

Figure6. Three-dimensional reconstructed confocal microscopy images of giant vesicles containing biotin-functionalized phosphol ipid and incubated with 1:1 streptavidin/Texas-Red avidin mixtures. The crystals of unlabeled streptavidin appear as black domains surrounded by fluorescent noncrystall izable avidin. Fivedifferent vesi cles are shown toillustratethe various crystal domains. Scale bar represents $10 \mu \mathrm{m}$.
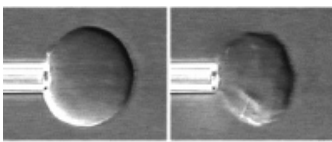

$\mathbf{t}=\mathbf{0} \mathrm{s}$

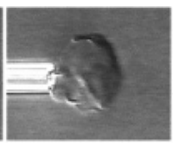

$\mathrm{t}=\mathbf{0 . 4 \mathrm { s }}$

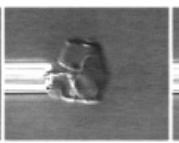

$\mathrm{t}=\mathbf{0 . 6 \mathrm { s }}$

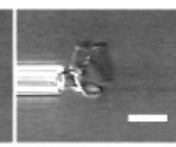

$\mathrm{t}=\mathbf{0 . 8 \mathrm { s }}$

Figure 7. Video image sequence of the rupturing process of streptavidin-coated vesicles showing a slow crumpling behavior and the presence of sharp edges on the surface. Scale bar represents $10 \mu \mathrm{m}$.
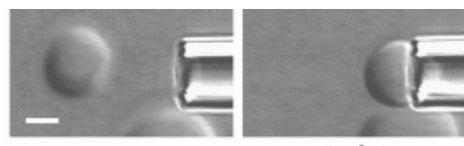

$\mathrm{t}=0 \mathrm{~s}$

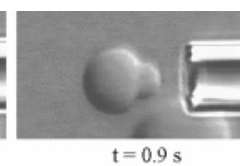

$\mathrm{t}=0.9 \mathrm{~s}$

Figure8. Videoimagesequence of a streptavidin-coated vesicle being partially aspirated into a micropi pet and quickly released. The persistent deformation after release indicates plastic deformation. Scale bar is $5 \mu \mathrm{m}$.

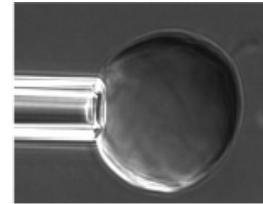

$39 \mathrm{~Pa}$

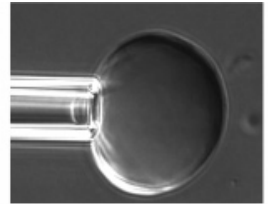

$107 \mathrm{~Pa}$

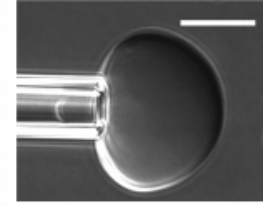

$235 \mathrm{~Pa}$

Figure 9. Shapechanges of a streptavidin-coated vesicleupon aspiration. The numbers indicate the suction pressure inside the micropi pet. At higher suction pressures, the membranes shows wrinkles near the pipet, and the vesicle takes on a nonspherical shape. The shape is maintained until rupture at greater than $3000 \mathrm{~Pa}$. Scale bar is $20 \mu \mathrm{m}$.

and relaxation periods were applied to the vesicles. A representative profile of the vesicle projection length vs pressure is shown in Figure 11.

In general, when a pressure pulse under $1000 \mathrm{~Pa}$ is applied, the responseis almost instantaneous. The vesicle projection remains after the suction pressure is reduced, and nosignificant increasein length occurs if a subsequent pressure pulse of the same magnitude is applied. When a suction pressure above the threshold (approximately $1000 \mathrm{~Pa}$ ) is applied, the vesicle responds with a slower exponential viscoelastic behavior. When the pressure is subsequently reduced, thevesicle projection only partially recovers, and usually a slight increase in the permanent projection length is observed.

Wealso performed mobility tests to qualitatively study the fluidity of the lipid bilayer compared to that of the outer streptavidin shell. The test was done by selectively incorporating the corresponding layer with a fluorescent dye, which was then bleached and the fluorescence recovery was monitored. Figure 12 shows thefluorescence recovery of a bleached area on a streptavidin-coated vesicle in which fluorescent NBD-PC lipid was incor porated into the lipid bilayer.

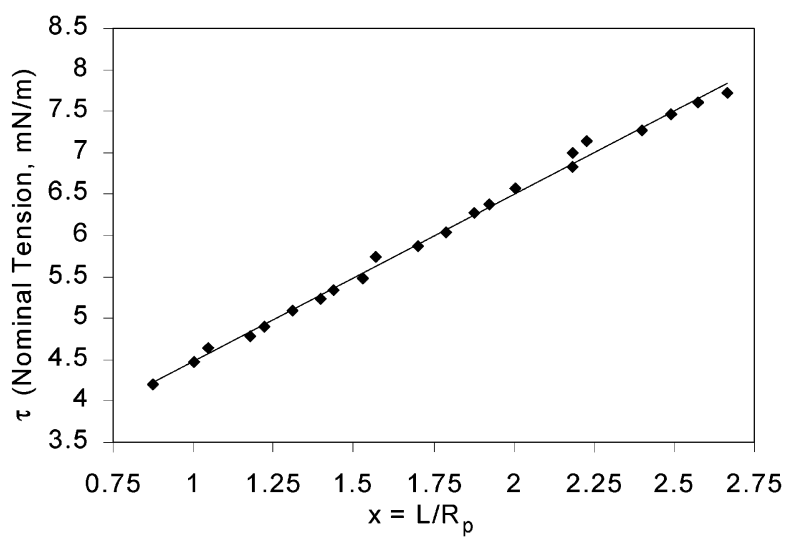

Figure 10. Typical plot of nominal tension $\tau$ vs dimensionless projection length $x=L / R_{p}$ for streptavidin-coated vesicles. The vesicle projection length increases linearly with the applied suction pressure.

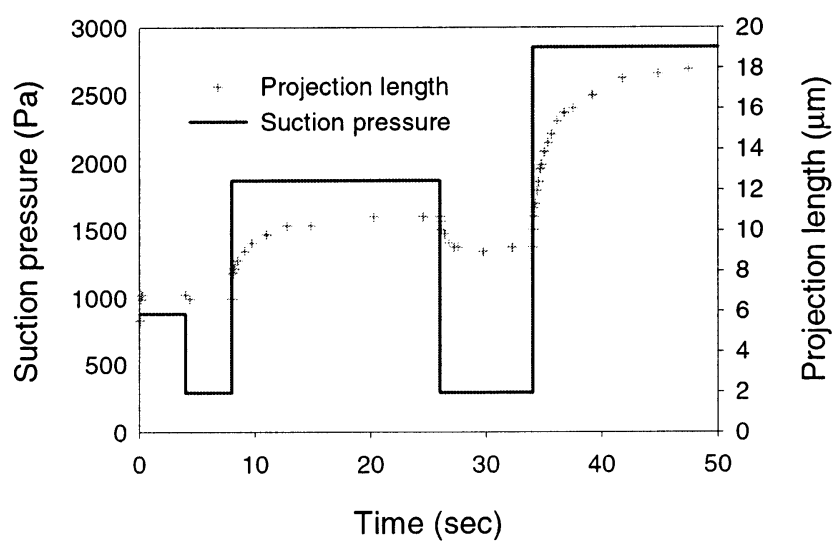

Figure 11. Representative response profile of streptavidincoated vesicles to alternating pressure pulse and relaxation periods. The + symbols represent thevesicle projection lengths, while the sol id line marks the applied suction pressures being applied. The diameters of the vesicle and the pipet in this particular experiment are 29.5 and $6.6 \mu \mathrm{m}$, respectively.

The lipid fluorescence in the photobleached region recovers fairly rapidly. The recovery rate is comparable tothat of barevesicles (barevesicles recover approximately twice as fast, data not shown). When the streptavidin layer is sel ectively label ed and photobleached, on theother hand, the fluorescence did not recover over a period of 153 $\mathrm{s}$, indicating the solidlike nature of the crystalline shell (Figure 13).

By selectively photobleaching the two layers, we observed that despite the strong coupling between the streptavidin crystals and the biotin-functionalized lipid bilayer, the lipid bilayer remains fluid while the streptavidin layer is immobilized as a polycrystalline shell. 
A)

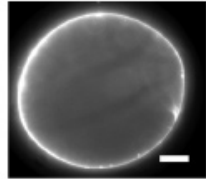

B)
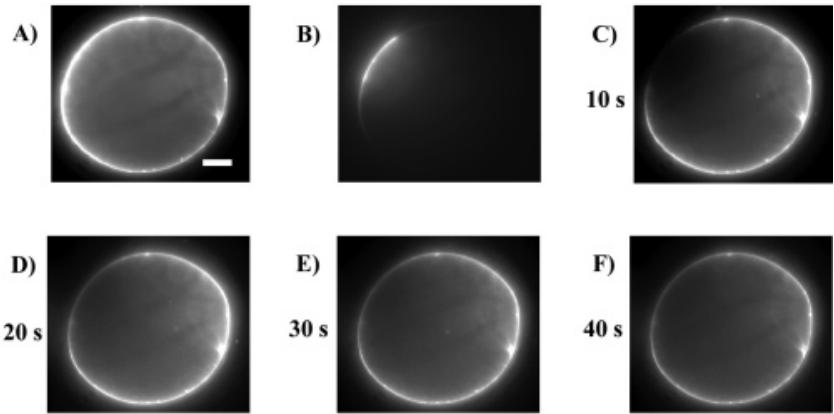

Figure 12. Fluorescence recovery of a photobleached region on a streptavidin-coated vesicle where fluorescent NBD-PC phospholipid molecules were incorporated in the lipid bilayer. (A) The initial streptavidin-coated vesicle. (B) Photobleaching of the upper left corner of the vesicle. $(C-F)$ Series of images showing the fluorescence recovery of the lipid layer. The bleached edge on the upper left side of the vesicle was clearly visible in less than $60 \mathrm{~s}$. Scale bar is $10 \mu \mathrm{m}$.
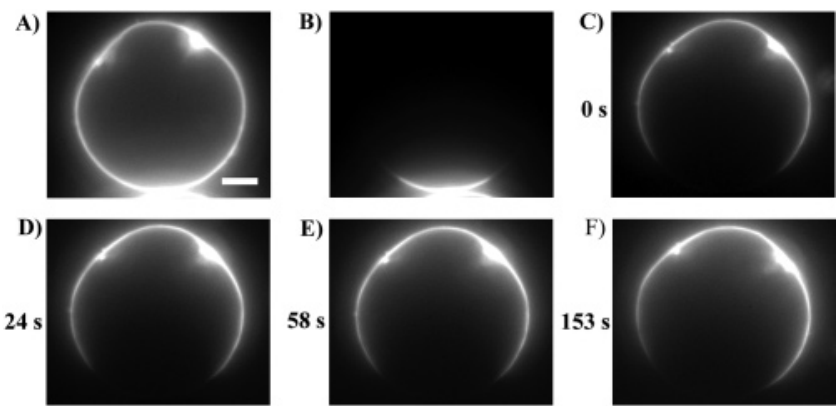

Figure 13. Fluorescence recovery of a photobleached region on a streptavidin-coated vesicle where the streptavidin molecules werefluorescently label ed with Texas-Red. (A)Theinitial streptavidin-coated vesicle. (B) Photobleaching thebottom area on the vesicle. (C-F) Sequential images following the photobleaching. The bleached vesicleedgewas nolonger visible. Scale bar is $5 \mu \mathrm{m}$.

\section{Discussion and Analysis}

When the bare vesicles were incubated with either avidin or streptavidin, the protein layer suppressed the thermal undulations and madethemembranesmorerigid. Thetightly bound protein layer reduces li pid mobility and produces a viscoelastic response to expansion. In conjunction with therelatively largesize of the protei n molecules, protein-protein and protein-lipid interactions contribute to the reduction of membrane flexibility.

Avidin-coated giant bilayer vesicles have been studied by $\mathrm{N}$ oppl-Simon and Needham in the context of vesicle adhesion and cross-bridgeformation. ${ }^{42}$ Theeffect of avidin on the elastic moduli of gaint vesicles has not been reported. Avidin molecules on the vesicle surface behave as a two-dimensional liquid, and the lateral interactions between them are weaker than those in streptavidin. Mean-field analysis indicates a crystallization energy of approximately $10 \mathrm{k}_{\mathrm{B}} \mathrm{T}$, where $\mathrm{k}_{\mathrm{B}}$ is the B oltzmann constant and $T$ is the absolute temperature. ${ }^{43}$ Although the energetic difference is not large, its effect on the vesicle properties is quite pronounced. The avidin-coated vesicles show characteristics similar tothose of bare vesicles, such as the spherical shape and elastic recovery after deformation, and similar values for the area expansion modulus. The data for the area expansion modulus is more widely

(42) Noppl-Simson, D. A.; Needham, D. Biophys. J . 1996, 70, 13911401.

(43) Coussaert, T.; Völkel, A. R.; Noolandi, J .; Gast, A. P. Biophys. J . 2001, 80, 2004-2010. scattered than that for bare vesicles dueto the noticeable affinity of the avidin-coated vesicles to the glass pipet, as shown in Figure 3B. The slow rupturing of the avidincoated vesicles indicates an increased dissipation caused by the avidin layer on the surface.

Streptavidin-coated vesiclesshow dramatically different properties from those of bare and avidin-coated vesicles. It is obvious from Figure 6 that streptavidin crystallites are present on the surface, and are responsible for the unique behavior seen. As Figure 5 shows, two typical vesicle morphologies are observed. Confocal microscopy images show that streptavidin crystallites on the vesicle surface tend to exhibit strong shape anisotropy due to a preferential growth direction. This effect is more pronounced at higher $\mathrm{pH}$ values. ${ }^{27}$ Such shape anisotropy in conjunction with parallel growth directions of multiple crystallites to minimize the overall bending energy can lead to the distortion of the vesicles al ong one axis.

Perhaps the most distinct characteristic of the streptavidin-coated vesicles is the rapid permanent deformation followed by slow viscoelastic relaxation. Under the specified experimental conditions, thetransition point between these two responses occurs at approximately $1000 \mathrm{~Pa}$ of suction pressure or at approximately $2 \mathrm{mN} / \mathrm{m}$ of tension. In most cases, even a small suction pressure of $300 \mathrm{~Pa}$ is sufficient to form a permanent projection.

Permanent (plastic) deformation behavior has been reported in biol ogi cal membranes such as red blood cell $s^{44}$ and, more recently, in polyelectrolyte microcapsules. ${ }^{45}$ Although streptavidin-coated vesicles are significantly simpler in composition, they arealso capable of producing plastic deformation.

The first indication of the underlying mechanism can be found in the apparent area expansion modulus of streptavidin-coated vesicles. The estimated values are significantly lower than thoseobtained from bareor avidincoated vesicles.

According to recent studies, the value for the Young's modulus for similar protein crystals such as lysozymeand ribonuclease fall in the range $0.5-17.5$ GPa. ${ }^{46}$ Based on these values, the analogous two-dimensional values of the area expansion modulus can be calculated by ${ }^{41}$

$$
\mathrm{K}=\frac{\mathrm{hE}}{2(1-v)}
$$

where h is the shell thickness, E is the Young's modulus, and $v$ is Poisson's ratio. If we assume a value of $5 \mathrm{~nm}$ for the shell thickness and 0.5 for Poisson's ratio of an incompressible material, the approximate values of $\mathrm{K}$ range between $2.5 \times 10^{3}$ and $8.75 \times 10^{4} \mathrm{mN} / \mathrm{m}$. These values are at least an order of magnitude greater that those obtained from streptavidin-coated vesicles and are thefirst indications that thetwo-dimensional streptavidin crystallites remained relatively undeformed on the vesicle surface throughout the membrane deformation.

The presence of permanent deformation at very low suction pressures along with the absence of a detectable linear elastic regime and yield point suggest that the observed behavior is different from plastic deformations typically observed in other materials. From linear elasticity theory, we can estimate the material strain in a 5-nmthick elastic plate aspirated into a $4-\mu \mathrm{m}$ micropipet to

(44) Evans, E. A.; LaCelle, P. L. Blood 1975, 45, 29.

(45) Bäumler, H.; Artmann, G.; Voigt, A.; Mitlöhner, R.; Neu, B.; Kiesewetter, H. J . Microencapsul. 2000, 17, 651-655.

(46) Caylor, C. L.; Speziale, S.; Kriminski, S.; Duffy, T.; Zha, C.-S. Thorne, R. E. J . Crystal Growth 2001, 232, 498-501. 
protrude $0.5 \mu \mathrm{m}$ of less than $0.25 \% .{ }^{47}$ In our experiments we observe plastic behavior at those small deformations. This estimate implies that a streptavidin-coated membranehas an exceptionally low yield point for plastic fl ow.

Experimental evidence suggests that the streptavidincoated vesicle can be represented by a two-layer spherical shell model. Theouter layer is a rigid polycrystal lineshell attached to the underlying lipid bilayer by biotinconjugated phosphol ipid anchors. At the experimental $\mathrm{pH}$ of 5.0, the charge density of streptavidin on the biotinbinding face is essentially zero, ${ }^{48}$ thus the net electrostatic interactions with the neutral SOPC lipids should be minimal. Streptavidin is also known to have negligible nonspecific binding to neutral phospholipids, as evident from el lipsometric measurements ${ }^{49}$ and purely repulsive force-distance profiles between streptavidin and bare neutral phospholipid monolayers obtained by using the surface forceapparatus. ${ }^{50}$ As the crystal domains grow on the veside surface, small amounts of noncrystalline streptavidin are concentrated in regions between crystalline domains and prevent further crystallization. When thevesicles wereplaced in themanipulation chamber filled with a hyperosmotic glucose solution, osmotic shrinkage occurs; however, due to the tightly packed crystallites, the vesicles could not take on thediverse shapes normally observed under such conditions. ${ }^{37,51}$ This results in corrugations in the underlying lipid bilayer at the noncrystalline areas between crystalline domains (F igure 14A). DIC microscopy images of streptavidin vesicles support this model, as numerous creases and ridges are visible from the surface of vesicles in this state.

When a small amount of suction is applied tothe vesicle using a micropipet, the corrugations are merely straightened out (Figure 14A-B). Since the deformation involves simply the smoothing of the corrugations by aspirating parts of the excess bilayer area into the pipet without significant area dilation, the projection remains rigid and permanent. Aspiration of Texas-Red streptavidin-coated vesicles confirms that both the protein and the excess lipid bilayer are aspirated into the pipet. Since the major corrugations occur between grain boundaries, viscous dissipation due to lipid flow is negligible in this region, and the vesicles respond rapidly in this regime. After release of the suction pressure the lipid bilayer exerts negligible force on the polycrystalline shell.

Asthesuction pressureincreases, this process continues until the major corrugations have been extended (Figure 14C). At the transition point, further aspiration of the vesicleresults in area expansion of the underlying bilayer membrane, and the elastic properties of the bilayer dominate. In addition to the immobilized biotin-X DHPE pillars hindering movement of other lipid molecules, lipid flow against a rigid surface produces hydrodynamic friction. ${ }^{52}$ The two effects create significant dissipation in the bilayer, resulting in the observed exponential viscoelastic response of the membrane. The membrane area shrinks again following therelease of aspiration pressure. Therefore the lipid bilayer exerts a restoring force on the

(47) Landau, L. D.; Lifschitz, E. M. Theory of Elasticity; Pergamon Press: London, 1959; Vol. 7.

(48) Sivasankar, S.; Subramaniam, S.; Leckband, D. Proc. Natl. Acad. Sci. U.S.A. 1998, 95, 12 961-12 966.

(49) Reiter, R.; Motschmann, H.; Knol I, W. Langmuir 1993, 9, 2430 2435.

(50) Sheth, S. R.; Leckband, D. Proc. Natl. Acad. Sci. U.S.A. 1997 94, 8399-8404

(51) Dobereiner, H. G.; Kas, J .; Noppl, D.; Sprenger, I.; Sackmann, E. Biophys. J . 1993, 65, 1396-1403.

(52) Merkel, R.; Sackmann, E.; Evans, E.J . Phys. (Paris) 1989, 50 1535-1555.
$\mathbf{A}$
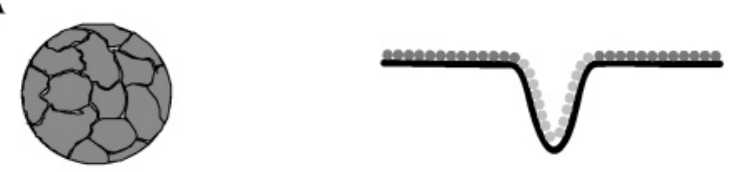

B
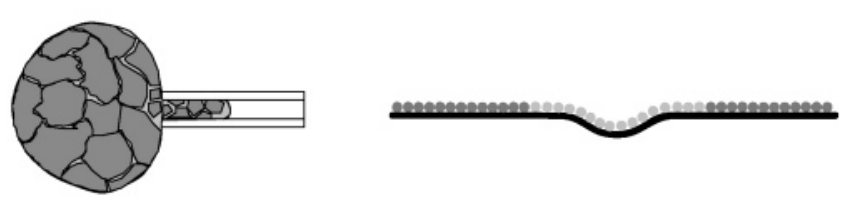

C
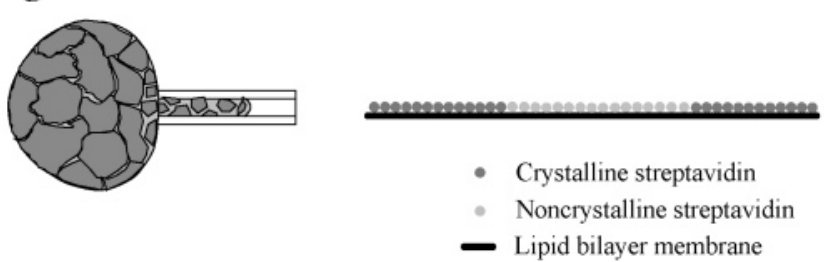

Figure 14. Schematic drawing of streptavidin crystals on the surface of a bilayer vesicle subjected to micropipet aspiration (not drawn to scale). (A) Osmotic shrinkagealong with the high rigidity of streptavidin crystals produces corrugations in the lipid bilayer. Micropipet aspiration of the vesicles in this state stretches out the corrugations at constant bilayer surface area. (B) Theprocess continues until major corrugations arestretched, after which the bilayer membrane tension begins to increase. (C) Further aspiration of the vesicle leads to surface area expansion of the lipid bilayer, and the elastic properties of the bilayer membranedominate. Thepolycrystallineshell remains relatively intact throughout most of the vesicle surface.

polycrystallineshell, which is responsiblefor theobserved elastic behavior in the high-tension regime.

The fate of the streptavidin crystallites during these processes can be deduced from the equilibrium shape of the aspirated vesicle. Figure 9 shows that the veside remains nonspherical during most of the aspiration process due to the persistence of largecrystallinedomains at the surface of the vesicle. Thus, with exception of areas in the vicinity of the pipet tip, the crystal network should remain relatively intact, and area dilation of the lipid bilayer should occur without significant perturbation to the outer protein layer.

Toaccount for theapparently low area expansion moduli of streptavidin-coated vesicles relative to those of bare or avidin-coated vesicles, thetwo-layer compositemembrane can be thought of as a Hookean element with two elastic springs in series. The first spring represents the elastic response of the lipid bilayer, while the second spring results from the protein crystals. Streptavidin crystals exist as flat rigid patches on the vesicl esurface and do not easily conform to the curvature of thebilayer surface. The vesicle thus appears to have many edges and facets. At increasing tensions, the facets are progressively smoothened out as the surface area of the membrane increases. Connecting the two elements in series results in a combined spring constant that is smaller than that of the individual elements. Sincethe extent of total area dilation depends also on thenature of the polycrystallinestructure, inhomogeneity in the crystalline shell causes a greater variation in the values of the measured moduli.

As we have shown, streptavidin-coated vesicles show combined properties of both thestreptavidin crystals and the fluid lipid bilayer to yield thin, rigid, yet viscoelastic composite membrane. In contrast to their rigid appearance, the existence of a polycrystalline shell does not 
increase the toughness of the vesicles. The toughness, or cohesi veenergy density, can beestimated from theintegral of the tension with respect to areal strain up to the point of rupture. ${ }^{53,54}$ Streptavidin-coated vesicles have a toughness of $0.11 \pm 0.05 \mathrm{~mJ} / \mathrm{m}^{2}(\mathrm{n}=15)$, within error of thevalue obtained from bare SOPC vesicles. ${ }^{39}$ Thus under hightension, streptavidin-coated vesicles show elastic properties of the underlying lipid bilayer, while retaining the rigid characteristics of a polycrystalline shell. Certain characteristics including the rigid appearance, vesicle shape transition during aspiration, and the formation of wrinkles are strikingly similar to those found in crosslinked block copolymer vesicles; ${ }^{40}$ however, they do not see the more complex behavior seen here such as plastic deformation and viscoelasticity due to their simpler membrane composition.

The experimental data presented above clearly show that theextent of protein-lipid interaction influences the properties of the composite membrane. In the case of most bacterial surface layers, the s-layer proteins are known to interact with the lipid bilayer via strong electrostatic interactions. Such interactions would surely involve the majority of lipid molecules under the protein layer. The situation is different with streptavidin-coated vesicles, where strong electrostatic interactions between neutral SOPC phospholipids and streptavidin molecules werenot present, and the majority of interactions occur by the biotin-streptavidin ligand-receptor interaction. ${ }^{49} \mathrm{E}$ ach streptavidin molecule is pinned to the bilayer by at most two biotin-functionalized phospholipids, whiletherest of the lipid molecules remain relatively independent of the

(53) Callister, W. D. Materials Science and Engineering: An Introduction; Anderson, W., Ed.; J ohn Wiley \& Sons: New York, 2000.

(54) Discher, B. M.; Won, Y.-Y.; Ege, D. S.; Lee, J . C.-M.; Bates, F. S.; Discher, D. E.; Hammer, D. A. Science 1999, 284, 1143-1146. protein layer. Despite this minimal interaction with the lipid layer, streptavidin-coated vesicles show significant morphological and mechanical changes with protein ordering.

\section{Conclusion}

Through the use of micropipet manipulation and microscopy, we studied the effects of a monomolecular layer of avidin or streptavidin on giant lipid bilayer vesicles. Binding of noncrystallizableavidin increased the bilayer bending rigidity and greatly suppressed thermal undulations. Theelastic responseto dilation of thevesicles was not significantly altered over that of bare vesicles. When the vesicles were coated with streptavidin, more complex behavior resulted. In addition to the dramatic reduction in thermal undulations seen with avidin-coated vesicles, the streptavidin crystallizes into a two-dimensional polycrystallineshell. The crystal morphology along with the high bending rigidity of the crystallites transformed the vesicles into roughened spheres and prolate ellipsoids. The composite protein-lipid membrane combines the solid characteristics of two-dimensional crystals with the fluid elastic behavior of lipid bilayers to yield a thin, rigid, yet viscoelastic structure. The nature of the protein-lipid and protein-protein interactions plays a key role in determining the overall properties of the composite membrane.

Acknowledgment. This work was supported by NASA Grant NAG3-2398 and the grant Me1458/3 by the Deutsche Forschungsgemeinschaft (DFG).

\section{LA026251Y}

